\title{
On the elastic driving force in diffusion-induced grain boundary motion
}

\author{
Oliver Penrose
}

Department of Mathematics, Scott Russell Building

Heriot-Watt University, Riccarton, Edinburgh EH14 4AS, Scotland, UK.

Keywords: diffusion-induced grain boundary motion (DIGM), interface dynamics, modelling, elastic behaviour, elastic misfit

\begin{abstract}
In 1983 Hillert obtained the formula $Y \eta^{2}\left(c_{+}-c_{-}\right)^{2}$ for the driving force per unit area of grain boundary arising from elastic misfit in an isotropic alloy, where the mole fractions $c_{+}$and $c_{-}$on the two sides of the grain boundary are small, $\eta$ is a measure of the elastic misfit and $Y=E /(1-\nu)$ where $E$ is Young's modulus and $\nu$ is Poisson's ratio. It is shown here that the formula is still valid (with suitably defined $Y, \eta$ ) when $c_{+}, c_{-}$are not small. The formula for $Y$ in a general anisotropic solid is given. The physical origin of the elastic force on the grain boundary is considered, with help from the 'energy-momentum tensor' devised by Eshelby to quantify the forces on other crystal imperfections such as dislocations. The theory also makes a prediction about the direction of motion of an initially stationary grain boundary.
\end{abstract}

\section{Introduction}

When a thin polycrystalline film of a metal is placed in a vapour consisting of another metal, it can happen that some crystal 
grains grow whilst others shrink, while atoms from the vapour diffuse in along the grain boundaries and are left behind in the newly-formed parts of the growing crystal grains. This phenomenon of diffusion-induced grain-boundary motion (DIGM), first demonstrated by Hillert and Purdy [1] in Fe-Zn, has since been observed in many other alloy systems: for reviews see $[2,3]$. There is no general agreement about the nature of the force that drives DIGM the various proposals are briefly reviewed in $[2,4,5]$ - but there is experimental evidence $[3,6,7]$ that, in some cases at least, an elastic driving force predominates.

The elastic mechanism appears to have been first proposed in about 1960 by Sulonen [8] who says [9], "A zone, impoverished or enriched in solute ... is formed ahead of the .. interface as a result of rapid grain boundary diffusion .... . This zone ... is subjected to ... lattice misfit stresses... It is the lattice strain energy that gives rise to the driving force ... because there is no counterpart ... on the side of the new matrix". A quantitative theoretical treatment of this mechanism was given in 1983 by Hillert [10]. He found that the driving force per unit area of grain boundary in an elastically isotropic material is *

$$
\frac{E \eta^{2}}{(1-\nu)}\left(c_{+}-c_{-}\right)^{2}
$$

where $E$ is Young's modulus, $\nu$ is Poisson's ratio, $\eta$ is the relative change in lattice spacing per unit change in solute mole fraction, and $c_{+}, c_{-}$(assumed by Hillert to be small) are the solute mole fractions well in front of and well behind the grain boundary. The physical origin of the formula (1) is that it is the elastic energy per unit volume at a place where the solute mole fraction differs by $c_{+}-c_{-}$from its value at zero stress; this energy formula was derived (for a case with sinusoidal composition dependence) by Cahn [11].

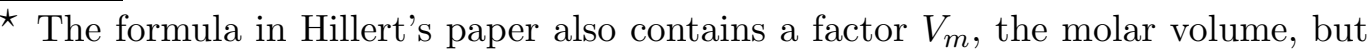
this factor appears to be due to the oversight of equating the force to a difference in molar free energies rather than free energies per unit volume.
} 
In Hillert's theory the grain boundary is treated as a very thin layer of liquid, both faces of which are in equilibrium with the neighbouring crystal. However, as pointed out in ref. [2], this assumption imposes too many constraints, since it requires the chemical potentials of both species of atom to be continuous at the interface. Hillert avoids the difficulty by requiring only the solute chemical potential to be continuous, but in order to treat alloys of arbitrary composition one would like to have a theory that treats both species of atom in the same way. In the present theory, there is no liquid layer and no phase equilibrium, and so neither chemical potential need be continuous; what does have to be continuous is the diffusion potential of Larché and Cahn [12-14]. For interstitial alloys, the diffusion potential is equal to the solute chemical potential so that our continuity condition is equivalent to the one used by Hillert, but for substitutional alloys the diffusion potential is equal to the difference between the chemical potentials of the two species of atom. We shall show that, for either type of alloy, Hillert's formula (1) is a good approximation even when the solute mole fraction is not small.

The nature of the elastic driving force is quite subtle. Although it arises from elastic stresses, it is not an elastic force in the usual sense : even though the stress tensor is discontinuous across the grain boundary, its normal component is continous and the material is macroscopically in elastic (mechanical) equilibrium. The usual kind of elastic force acts on all the atoms an a given region in the same way, tending to change the local macroscopic configuration of the material, but the force considered here acts differentially, tending to change the microscopic configuration of the atoms in such a way that they fit the lattice of the growing grain rather than the shrinking one, but not changing the macroscopic configuration. It is analogous to the forces acting on dislocations and other crystal imperfections, discussed by Eshelby in his paper [15] on the elastic "energy-momentum" tensor. More detail about this application of Eshelby's tensor is given in section 7 . The magnitude of the force acting on the grain bound- 
ary will be evaluated by equating it to the decrease in total free energy (which is approximately the decrease in elastic energy) when the grain boundary advances through unit distance. If the grain boundary is curved, there will also be a capillary force, but in the present paper this effect will be neglected and the grain boundary will be treated as a plane ${ }^{\star \star}$.

Following Larché and Cahn [14], we shall treat the metal as an elastic continuum and model the diffusion in it by making the diffusional flux proportional to the gradient of the diffusion potential. The grain boundary is treated as a plane surface across which the diffusion potential is continuous. This continuity of diffusion potential does not, however, imply continuity of composition, because the diffusion potential depends also on the local elastic stress, which is not continuous across the grain boundary.

To justify these modelling assumptions, the grain boundary speed $v$ must not be too large nor too small. If $v$ is too large the solute does not diffuse far enough ahead of the grain boundary to justify the use of elastic continuum theory, so that the atomic structure of the metal must be allowed for; if $v$ is too small, the solute diffuses so far ahead of the grain boundary that the thickness of the specimen and/or the curvature of the grain boundary must be allowed for. In either case, the elastic mechanism may still be effective, but the treatment given here would be too simple. The range of values for $v$ over which our simple treatment should apply is made more precise in section 8, particularly eqn (47).

In 1997 Cahn et al. [5] proposed a phase-field model for DIGM which made possible a comparison of various different driving mechanisms. It was concluded that the elastic driving force was the best candidate, though perhaps not the only one. In that paper, however, the elastic energy changes were modelled in an

\footnotetext{
${ }^{\star \star}$ A mathematical model detailing the way in which the elastic driving force and the capillary force due to curvature of the grain boundary interact with diffusion in the grain boundary to determine the shape and velocity of the moving grain boundary is studied in refs [16],[17]. The elastic driving force seems also to be important for the theory of discontinuous preciptiation; see ref. [18].
} 
ad hoc way, while at the same time quite strong assumptions were made about the internal structure of the grain boundary. The model used here is quite different. It is not a phase-field model; the grain boundary is represented not as a layer in which the field variables vary continously but as a plane surface at which they change discontinuously. Moreover the diffusivity in the crystal grains is not neglected as it was in [5]; indeed it will play an essential part.

\section{The elastic energy and equilibrium conditions}

The Helmholtz free energy of the specimen can be written $\star \star \star$

$$
F:=\int_{\Omega}\left[f_{0}(c(\mathbf{x}))+w(\mathbf{x})\right] d^{3} \mathbf{x},
$$

where $f_{0}(c)$ is the free energy per unit volume at zero stress and solute mole fraction $c$, and $w$ is the elastic free energy per unit volume. The variable of integration $\mathbf{x}:=\left(x_{1}, x_{2}, x_{3}\right)$ is the position vector of the relevant material point in some uniform reference configuration of the specimen, so that a given material point always has the same value of $\mathbf{x}$, regardless of where the elastic deformation has taken it to. In this paper the reference configuration is taken to coincide with the configuration of the crystal far in front of the moving grain boundary. The region occupied by the specimen in the reference configuration is denoted by $\Omega$.

According to linear elasticity theory [20,21], the elastic free energy $w$ per unit reference-configuration volume is a quadratic function of the Cartesian components $u_{1}, u_{2}, u_{3}$ of displacement relative to the reference configuration:

$$
w=\frac{1}{2} \sum_{i, j, k, l} \lambda_{i j k l}(\Delta e)_{i j}(\Delta e)_{k l}
$$

$\bar{\star}$ External tractions, such as the ambient pressure, can be taken into account by adding another term to the formula (2). See, for example, eqn (10) of ref. [20]. 
where $\lambda_{i j k l}$ is the elastic stiffness tensor and $(\Delta e)_{i j}$ means $e_{i j}-$ $e_{i j}^{(s f)}$ where $e_{i j}:=\frac{1}{2}\left(\partial u_{i} / \partial x_{j}+\partial u_{j} / \partial x_{i}\right)$ is the strain relative to the reference configuration and $e_{i j}^{(s f)}$ is the stress-free strain, which depends on the local composition and on the local crystal structure. If the crystal axes in the two grains are differently oriented, $\lambda_{i j k l}$ will be different on the two sides of the grain boundary.

We shall model the dependence of stress-free strain on composition by assuming linearity (Vegard's law), i.e. $e_{i j}^{(s f)}=e_{i j}^{(0)}+(c-$ $\left.c_{+}\right) \eta_{i j}$, in which $c_{+}$is the solute mole fraction far in front of the grain boundary, $e_{i j}^{(0)}$ is the stress-free strain at solute mole fraction $c_{+}$, and the tensor $\eta_{i j}$ represents the misfit strain. In cubic crystals the misfit strain is isotropic, i.e. $\eta_{i j}=$ const. $\delta_{i j}$ where $\delta_{i j}$ is the unit tensor, but in hexagonal crystals it can be highly anisotropic - see ref. [22]. The stress-free strain $e_{i j}^{(0)}$ depends only on the crystal structure and its relation to the reference configuration. The value of $e_{i j}^{(0)}$ is different for each crystal grain, but is independent of position within the grain. In the shrinking grain, far in front of the grain boundary, the definition of the reference configuration ensures that the strain is zero; since this part of the grain is also stress-free and has $c=c_{+}$, it follows from the above 'Vegard' formula that $e_{i j}^{(0)}=0$ there, and hence that $e_{i j}^{(0)}=0$ throughout the shrinking grain. In the growing grain, $e_{i j}^{(0)}$ is still independent of position, but it is not zero. The reason for the difference is that, as the grain boundary passes by, the material recrystallizes, and the new crystal structure is related to the reference configuration in a different way. The rearrangement is equivalent to a plastic deformation without volume change (as in toothpaste squeezed out of its tube), so that $\sum_{i=1}^{3} e_{i j}^{(0)}=0$. The equation for $(\Delta e)_{i j}$ is therefore

$$
(\Delta e)_{i j}=e_{i j}-e_{i j}^{(s f)}=\frac{1}{2}\left\{\frac{\partial u_{i}}{\partial x_{j}}+\frac{\partial u_{j}}{\partial x_{i}}\right\}-\left\{e_{i j}^{(0)}+\left(c-c_{+}\right) \eta_{i j}\right\}
$$


where $e_{i j}^{(0)}=0$ in front of the grain boundary and $\sum_{i} e_{i i}^{(0)}=0$ behind.

The elastic strains are determined by minimizing the total free energy (2) with respect to the displacement field $\mathbf{u}(\mathbf{x})$, subject to certain constraints. The Euler-Lagrange equations for this minimization problem are the equations of elastic equilibrium $[20,21]$

$$
\sum_{j} \partial t_{i j} / \partial x_{j}=0
$$

where $t_{i j}$ is the stress tensor given by

$$
t_{i j}=\sum_{k l} \lambda_{i j k l}(\Delta e)_{k l} .
$$

We shall assume that elastic equilibrium is reached instantaneously, so that (5) holds at all times and at all places other than the grain boundary.

\section{The elasticity problem}

We represent the grain boundary as a plane, parallel to the $x_{1}, x_{2}$ plane in a suitable coordinate system, travelling in the positive $x_{3}$ direction at a constant positive velocity $v$. With a suitable choice for the time and space origins, the equation of the grain boundary at time $t$ is then $x_{3}=v t$. We assume that the solute mole fraction and the stresses and strains are independent of $x_{1}, x_{2}$ and that they approach limits (not necessarily the same) far in front of the moving grain boundary $\left(x_{3} \rightarrow+\infty\right)$ and far behind $\left(x_{3} \rightarrow-\infty\right)$. In particular, the stress is zero far from the grain boundary and the solute mole fractions $c_{+}, c_{-}$far from the grain boundary are

$$
\lim _{x_{3} \rightarrow \pm \infty} c\left(x_{1}, x_{2}, x_{3}, t\right)=c_{ \pm} .
$$

We shall look for a solution of the relevant elasticity problem in which $\mathbf{u}:=\left(u_{1}, u_{2}, u_{3}\right)$ is a function of $x_{3}$ only, continuous 
everywhere and differentiable except at the grain boundary. In front of the grain boundary, we have $e_{i j}^{(0)}=0$, and (4) becomes

$$
(\Delta e)_{i j}=\frac{1}{2}\left\{\frac{d u_{i}}{d x_{3}} \delta_{3 j}+\frac{d u_{j}}{d x_{3}} \delta_{3 i}\right\}-\Delta c \eta_{i j} \quad\left(x_{3}>v t\right)
$$

where $\Delta c:=c-c_{+}\left(x_{3}>v t\right)$. The stress tensor, according to (6), is therefore

$$
t_{i j}=\sum_{k} \lambda_{i j k 3} d u_{k} / d x_{3}-\Delta c \sum_{k} \sum_{l} \lambda_{i j k l} \eta_{k l} \quad\left(x_{3}>v t\right)
$$

where we have used the symmetry relation $\lambda_{i j k 3}=\lambda_{i j 3 k}$.

The equation of elastic equilibrium (5) simplifies here to $\partial t_{i 3} / \partial x_{3}$ $=0$; the value of $t_{i 3}$ is therefore equal to its value at $+\infty$, which is zero. It follows, on setting $j=3$ in (9), that

$$
0=\sum_{k} \lambda_{i 3 k 3} d u_{k} / d x_{3}-\Delta c \sum_{k} \sum_{l} \lambda_{i 3 k l} \eta_{k l}\left(i=1,2,3 ; x_{3}>v t\right)(10)
$$

This is a system of three linear equations for the three unknowns $d u_{k} / d x_{3}(k=1,2,3)$. Its solution can be written

$$
d u_{i} / d x_{3}=\Delta c \sum_{k} \sum_{m} \sum_{n} Z_{i k} \lambda_{k 3 m n} \eta_{m n}
$$

where $Z_{i k}$ denotes the inverse of the matrix $\left(Z^{-1}\right)_{i k}:=\lambda_{i 3 k 3}$. Substituting (11) into (8) and the result into (3) we find that

$$
w=Y(\eta \Delta c)^{2} \quad\left(x_{3}>v t\right)
$$

where by definition $\eta:=\frac{1}{3} \sum_{i} \eta_{i i}$ and

$$
Y:=\frac{1}{2 \eta^{2}}\left[\sum_{i, j, k, l} \lambda_{i j k l} \eta_{i j} \eta_{k l}-\sum_{i, j, k, l, m, n} \lambda_{i 3 j k} \eta_{j k} Z_{i l} \lambda_{l 3 m n} \eta_{m n}\right]
$$

It is a fortunate consequence of the special geometrical symmetry assumed here (solutions depending only on $x_{3}$ ) that even with 
anisotropic elasticity the free energy density $w$ depends only on the local value of $c$, i.e. the one at the same $x_{3}$.

The formula (13) is easily generalized to the case where the normal to the grain boundary lies not along the third coordinate axis but along an arbitrary unit vector $\mathbf{n}=\left(n_{1}, n_{2}, n_{3}\right)$; since $\lambda_{i j k l}$ is a tensor, the generalization is

$$
Y(\mathbf{n}):=\frac{1}{2 \eta^{2}}\left[\sum_{i j k l} \lambda_{i j k l} \eta_{i j} \eta_{k l}-\sum_{i j k l m n p q} n_{p} \lambda_{i p j k} \eta_{j k} Z_{i l}(\mathbf{n}) n_{q} \lambda_{l q m n} \eta_{m n}\right]
$$

where $\left[Z(\mathbf{n})^{-1}\right]_{i k}=\sum_{p, q} \lambda_{i p k q} n_{p} n_{q}$.

The expression (14), for the isotropic misfit case $\eta_{i j}=\eta \delta_{i j}$, appears in the theory of structural transformations in solids [23] and in the theory of phase separation in alloys with coherent elastic misfit (eqn (31) of ref. [20]). For isotropic elasticity and misfit, eqn (14) reduces to $Y=E /(1-\nu)$ where $E$ is Young's modulus and $\nu$ is Poisson's ratio, a formula due to Cahn [11]; Cahn also gave an approximate generalization to anisotropic cubic crystals [24]. The formula for $Y$ in the case of isotropic misfit but anisotropic elasticity was given by Khachaturyan [25], in a calculation of the energy of a crystal with space-dependent composition; an equivalent formula was obtained independently by Hilliard [26]. A case with anisotropic misfit (a Zn-Cd alloy) is considered in ref.[22], although the method used there appears to neglect the elastic strain term $e_{i j}$ in the formula (4) for $(\Delta e)_{i j}$.

To use (4) behind the grain boundary, define modified displacements $\tilde{u}$ by

$$
u_{i}:=\tilde{u}_{i}+\alpha\left(x_{3}-v t\right) \delta_{3 i}
$$

where $\alpha$ is a constant to be chosen so that $\tilde{\mathbf{u}}=0$ far behind the grain boundary (i.e. $\lim _{x_{3} \rightarrow-\infty} \tilde{\mathbf{u}}=0$ ); then (4) gives 


$$
(\Delta e)_{i j}=\frac{1}{2}\left\{\frac{d \tilde{u}_{i}}{d x_{3}} \delta_{3 j}+\frac{d \tilde{u}_{j}}{d x_{3}} \delta_{3 i}\right\}+\alpha \delta_{3 i} \delta_{3 j}-\left(c-c_{+}\right) \eta_{i j}-e_{i j}^{(-)}
$$

where $e_{i j}^{(-)}$means the value of $e_{i j}^{(0)}$ for $x<v t$. In the limit $x_{3} \rightarrow$ $-\infty$, both $\tilde{\mathbf{u}}$ and $t_{i j}$ are zero so that, by (6), eqn (16) becomes

$$
0=\alpha \delta_{3 i} \delta_{3 j}-\left(c_{-}-c_{+}\right) \eta_{i j}-e_{i j}^{(-)}
$$

(whence $\alpha=3 \eta\left(c_{-}-c_{+}\right)$, since $\sum e_{i i}^{(-)}=0$ ). Subtracting, we find

$$
(\Delta e)_{i j}=\frac{1}{2}\left\{\frac{d \tilde{u}_{i}}{d x_{3}} \delta_{3 j}+\frac{d \tilde{u}_{j}}{d x_{3}} \delta_{3 i}\right\}-\Delta c \eta_{i j} \quad\left(x_{3}<v t\right)
$$

where $\Delta c:=c-c_{-}\left(x_{3}<v t\right)$. Eqn (18) is formally the same as (8) but with $\mathbf{u}$ replaced by $\tilde{\mathbf{u}}$, and the consequent elastic equilibrium calculation is likewise the same; so the formula for the elastic energy is still (12), but with $Y$ and $\Delta c$ now given their definitions for the growing grain (i.e. for $x_{3}<v t$ ).

\section{A diffusion problem}

If a small isothermal change $\delta c(\mathbf{x})$ is made in the composition field, and the displacement field $\mathbf{u}(\mathbf{x})$ changes so as to maintain elastic equilibrium, the changes in $\mathbf{u}(\mathbf{x})$ have no first-order effect on the free energy, so that the first-order change is, by (2), simply

$$
\int_{\Omega}\left[d f_{0} / d c+\partial w / \partial c\right] \delta c(\mathbf{x}) d^{3} \mathbf{x}
$$

where $\partial w / \partial c$ denotes the derivative of $w$ at constant strain.

Assuming for simplicity that the stiffness tensor $\lambda_{i j k l}$ does not depend on $c$, we have from (3), (4) and (6)

$$
\partial w / \partial c:=\sum_{i, j, k, l} \lambda_{i j k l}(\Delta e)_{i j}\left(-\eta_{k l}\right)=-\sum_{k, l} t_{k l} \eta_{k l} .
$$


The local diffusion potential $\mu(\mathbf{x})$ can be defined as the coefficient of $\delta c(\mathbf{x})$ in (19), that is

$$
\mu(\mathbf{x})=\mu_{0}(c(\mathbf{x}))+\partial w / \partial c=\mu_{0}(c(\mathbf{x}))-\sum_{k, l} t_{k l} \eta_{k l}
$$

where $\mu_{0}(c):=d f_{0}(c) / d c$ is the stress-free diffusion potential. The formula (21) (in the case of isotropic misfit) is due to Larché and Cahn [14], although their derivation is a little different. To evaluate the diffusion potential, we use (12) to evaluate the term $\partial w / \partial c$ in $(21)$, obtaining

$$
\mu=\mu_{0}(c)+2 \eta^{2} Y \Delta c .
$$

The formula (22) was given (for isotropic elasticity) by Larché and Cahn. The definition (21) is general, but (22) depends on our geometrical symmetry assumption that the composition and strain do not depend on $x_{1}$ and $x_{2}$.

The equation for diffusion in an elastic medium, as formulated by Larché and Cahn, is (since we are assuming $\partial / \partial x_{2}=\partial / \partial x_{3}=0$ )

$$
\frac{\partial c}{\partial t}=\frac{\partial}{\partial x_{3}}\left(B(c) \frac{\partial \mu}{\partial x_{3}}\right)
$$

where $c$ is the mole fraction of solute and $B(c)$ is a mobility coefficient. The mobility is related to the bulk diffusivity $D$ (which we assume to be independent of composition) by

$$
B(c)=D\left(\frac{d \mu_{0}(c)}{d c}\right)^{-1}
$$

so that (23) reduces to Fick's law $\partial c / \partial t=D \partial^{2} c / \partial x_{3}^{2}$ when $\mu=$ $\mu_{0}(c)$, i.e. when the stress is zero.

We look for a solution of (23) using the "travelling wave" ansatz

$$
c\left(x_{1}, x_{2}, x_{3}, t\right)=\phi\left(x_{3}-v t\right)
$$


where the function $\phi(x)$ is continuous except possibly at $x=0$, and satisfies the conditions $\phi(x) \rightarrow c_{ \pm}$as $x \rightarrow \pm \infty$. Substituting into (23) and using (24) we obtain an ordinary differential equation for $\phi$

$$
\begin{aligned}
-v \frac{d \phi(x)}{d x} & =\frac{d}{d x}\left(B(\phi(x)) \frac{d \mu(\phi(x))}{d x}\right) \\
& =D \frac{d}{d x}\left(\frac{\mu^{\prime}(\phi(x))}{\mu_{0}^{\prime}(\phi(x))} \frac{d \phi(x)}{d x}\right) \quad(x \neq 0)
\end{aligned}
$$

where $x$ means $x_{3}-v t, \mu(c)$ means the expression on the right side of (22), and the primes denote derivatives, so that $\mu^{\prime}(c)=$ $\mu_{0}^{\prime}(c)+2 Y \eta^{2}$.

Integrating (26) and using the condition (7), we obtain a firstorder differential equation which can be rearranged as

$$
\begin{array}{ll}
\frac{d \phi}{d x}=\left(\frac{v}{D}\right) \frac{c_{+}-\phi}{1+2 Y_{+} \eta^{2} / \mu_{0}^{\prime}(\phi)} & (x>0) \\
\frac{d \phi}{d x}=\left(\frac{v}{D}\right) \frac{c_{-}-\phi}{1+2 Y_{-} \eta^{2} / \mu_{0}^{\prime}(\phi)} & (x<0)
\end{array}
$$

where $Y_{ \pm}$denotes the value of $Y$ in the grain where $\pm x>0$. The quantity $\mu_{0}^{\prime}(\phi)$ is positive, by thermodynamic stability. For substitutional alloys this quantity can be estimated from ideal solution theory (according to which $\mu_{0}(c)=\left(R T / V_{m}\right) \ln [c /(1-c)]$, where $R$ is the gas constant per mole and $V_{m}$ is the molar volume) to be $\mu_{0}^{\prime}(\phi) \approx R T / V_{m}[c(1-c)]$, so that the correction term $2 Y \eta^{2} / \mu_{0}^{\prime}(\phi)$ in the denominator of $(27)$ is roughly $2 Y V_{m} c(1-$ c) $\eta^{2} / R T$. There is a similar formula for interstitial alloys. The correction is therefore small for dilute alloys, and since $\eta$ is generally fairly small one expects the term to be quite small even when the alloy is not dilute, although Larché and Cahn [14] give an example which illustrates how it can be as large as 0.2 . Their example uses $\eta=0.05, c=0.5$ and the elastic constants for the $\langle 111\rangle$ direction in copper. 
The boundary conditions on (27) are that $\phi(x) \rightarrow c_{ \pm}$as $x \rightarrow \pm \infty$ (by (7)) and that the diffusion potential $\mu(\phi)$ must be continuous at $x=0$. This last condition follows from our assumption that the grain boundary is thin enough to be modelled as a geometrical surface, so that there is a local equilibrium with respect to exchanges of atoms across it. On the other hand the mole fraction $c$ need not be continuous across the grain boundary, and there is in fact a small discontinuity there, which we calculate in the next section. Moreover, in contrast to most diffusion problems, there is no continuity condition on the normal component of mass flow (i.e. on the gradient of $c$ or of $\mu$ ) due to conservation of matter; this is because atoms can be emitted or absorbed by the grain boundary, the necessary matter being supplied or removed by diffusive transport within the grain boundary itself.

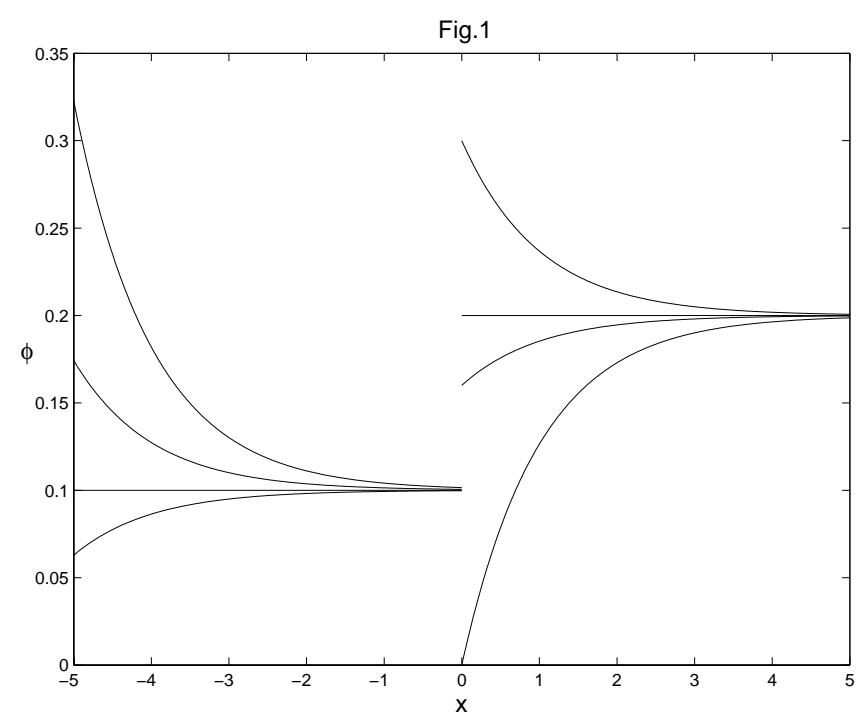

Fig. 1. Sample trajectories of the differential equation (27) for a case where $c_{-}=0.1, c_{+}=0.2$. The solutions need not be continuous at $x=0$. In the left-hand part of the diagram, where $x<0$ (i.e. $x_{3}<v t$ ), the only solution that is bounded on the entire half-line is the constant solution $\phi=c_{-}$; but in the right-hand part, where $x>0$, there are many bounded solutions.

Consider the trajectories of the differential equation (27) in the $(x, \phi)$ plane (see Fig. 1). They are in general discontinuous where they cross the abscissa $x=0$. There is one horizontal trajectory $\phi=c_{ \pm}=$const. on each side of this abscissa. Since the denominators in (27) are positive, the non-constant trajectories converge on the relevant constant trajectory as $x$ increases to $+\infty$, but 
diverge from it as $x$ decreases to $-\infty$. On the half-line $x<0$, the only bounded solution is the horizontal trajectory $\phi=c_{-}$; but on the half-line $x>0$ the solutions are all bounded, converging to $c_{+}$as $x \rightarrow+\infty$. In symbols, this conclusion can be written

$$
\begin{aligned}
& \phi(x)=c_{-} \quad(x<0) \quad \text { i.e. } c(\mathbf{x}, t)=c_{-} \quad\left(x_{3}<v t\right) \\
& \phi(x) \rightarrow c_{+} \text {as } x \rightarrow+\infty \text { i.e. } c(\mathbf{x}, t) \rightarrow c_{+} \text {as } x_{3} \rightarrow+\infty .
\end{aligned}
$$

The solute diffuses ahead of the grain boundary a distance of order $D / v$ (as noted already, in the non-elastic case, by Cahn and Balluffi [27]), but there is no diffusion behind the grain boundary.

\section{The matching condition at the grain boundary}

To complete the solution of (26) we apply the condition that the diffusion potential is to be continuous across the grain boundary.

$$
\mu(c(v t-0))=\mu(c(v t+0)),
$$

where $c(v t \pm 0):=\lim _{\varepsilon \backslash 0} c\left(x_{1}, x_{2}, v t \pm \varepsilon, t\right)$. Using (28) on the left side of (29) and (22) on both sides, we obtain

$$
\mu_{0}\left(c_{-}\right)=\mu_{0}(c(v t+0))+2 \eta^{2} Y_{+}\left(c(v t+0)-c_{+}\right)
$$

where $Y_{+}$is the value of $Y$ in front of the grain boundary, defined by using the elastic constants for the shrinking grain in (13).

An approximate solution for (30) can be found with the help of the mean-value theorem, which tells us that

$$
\mu_{0}\left(c_{-}\right)-\mu_{0}(c(v t+0))=\left[c_{-}-c(v t+0)\right] \mu_{0}^{\prime}(\bar{c})
$$

where $\mu_{0}^{\prime}(\bar{c}):=d \mu_{0}(\bar{c}) / d \bar{c}$ is evaluated at $\bar{c}$, a number lying somewhere between $c_{-}$and $c(v t+0)$. Using (31) in (30), we obtain 
$\left[c_{-}-c(v t+0)\right] \mu_{0}^{\prime}(\bar{c})=2 \eta^{2} Y_{+}\left(c(v t+0)-c_{+}\right)$. It follows, on solving this last equation for $c(v t+0)$, that

$$
c(v t+0)=\frac{c_{-} \mu_{0}^{\prime}(\bar{c})+2 \eta^{2} Y_{+} c_{+}}{\mu_{0}^{\prime}(\bar{c})+2 \eta^{2} Y_{+}}
$$

so that the total change in solute mole fraction across the shrinking grain is

$$
c(v t+0)-c_{+}=\frac{\mu_{0}^{\prime}(\bar{c})}{\mu_{0}^{\prime}(\bar{c})+2 \eta^{2} Y_{+}}\left(c_{-}-c_{+}\right) .
$$

The discontinuity in mole fraction at the grain boundary is

$$
\begin{aligned}
c(v t+0)-c(v t-0) & =c(v t+0)-c_{-} & \text {by }(28) \\
& =\frac{2 \eta^{2} Y_{+}\left(c_{+}-c_{-}\right)}{\mu_{0}^{\prime}(\bar{c})+2 \eta^{2} Y_{+}} & \text {by }(32) .
\end{aligned}
$$

\section{The force on the grain boundary}

The force on the grain boundary is the space rate of decrease of the total Helmholtz free energy when the grain boundary moves while the distribution of solute remains the same except just behind the grain boundary. Since the composition is discontinuous at the grain boundary, solute atoms must be supplied or removed as it passes by. These atoms enter or leave the crystal by travelling along the grain boundary, which will be represented here as a reservoir whose diffusion potential $\mu_{g b}$ equals the diffusion potential in the crystal at the place where it meets the grain boundary; this diffusion potential is the same for both crystals.

The total Helmholtz free energy is the sum of the free energy of the specimen and that of the reservoir. The free energy of the 
specimen per unit cross-section can be written, using (2), as

$$
\int_{-L}^{L}\left[f_{0}(c(\mathbf{x}))+w(\mathbf{x})\right] d x_{3},
$$

where $L$ is half the length of the crystal (assumed to be so large that we can take $c( \pm L)=c_{ \pm}$, etc.). The free energy of the reservoir is equal to a constant plus $\mu_{g b}$ times the number of solute atoms in it, and is therefore also equal to a constant minus $\mu_{g b}$ times the number of solute atoms in the specimen, which is $\int_{-L}^{L} c(\mathbf{x}) d x_{3}$ per unit cross-section. Thus the total Helmholtz free energy of crystal and reservoir together, per unit cross-section, is

$$
\begin{gathered}
\int_{-L}^{L}\left[f_{0}(c(\mathbf{x}))+w(\mathbf{x})-\mu_{g b} c(\mathbf{x})\right] d x_{3}+C \\
=\int_{-L}^{v t}\left[\varpi_{0}(c(\mathbf{x}))+w(\mathbf{x})\right] d x_{3}+\int_{v t}^{L}\left[\varpi_{0}(c(\mathbf{x}))+w(\mathbf{x})\right] d x_{3}+C(36)
\end{gathered}
$$

where $C$ is a constant and $\varpi_{0}(c):=f_{0}(c)-\mu_{g b} c$.

The force on the grain boundary is minus the space derivative of the free energy with respect to the grain boundary's position, which in the formula (36) is denoted by $v t$. Applying the fundamental theorem of calculus, we find that the force per unit area on the grain boundary in the positive $x_{3}$ direction is

$$
\begin{aligned}
p & :=-\left[\varpi_{0}(c(v t-0))+w(v t-0)\right]+\left[\varpi_{0}(c(v t+0))+w(v t+0)\right] \\
& =Y_{+} \eta^{2}\left[c(v t+0)-c_{+}\right]^{2}+\left[\varpi_{0}(c(v t+0))-\varpi_{0}\left(c_{-}\right)\right]
\end{aligned}
$$

where $w(v t \pm 0):=w\left(x_{1}, x_{2}, v t \pm 0, t\right)$. In the second line we have used (12) to evaluate $w(v t+0)$, and also (28), which tells us that $c(v t-0)=c_{-}$, with the consequence that $w(v t-0)=0$. Taylor's theorem (with remainder term) tells us that

$$
\varpi_{0}(c)=\varpi_{0}\left(c_{-}\right)+\left(c-c_{-}\right) \varpi_{0}^{\prime}\left(c_{-}\right)+\frac{1}{2}\left(c-c_{-}\right)^{2} \varpi_{0}^{\prime \prime}\left(\overline{\bar{c}}_{-}\right)
$$


where $\overline{\bar{c}}$ lies between $c_{-}$and $c$. The definitions of $\varpi_{0}, \mu_{0}$ and $\mu_{g b}$ give $\varpi_{0}^{\prime}\left(c_{-}\right)=f_{0}^{\prime}\left(c_{-}\right)-\mu_{g b}=\mu_{0}\left(c_{-}\right)-\mu_{g b}=0$ and $\varpi_{0}^{\prime \prime}(\overline{\bar{c}})=\mu_{0}^{\prime}(\overline{\bar{c}})$. Putting these expressions into (38), we can use it to write (37) as $p=Y_{+} \eta^{2}\left[c(v t+0)-c_{+}\right]^{2}+\frac{1}{2}\left[c(v t+0)-c_{-}\right]^{2} \mu_{0}^{\prime}(\overline{\bar{c}})$ with $\overline{\bar{c}}$ lying between $c_{-}$and $c(v t+0)$. It follows, by (33) and (34), that

$$
\begin{aligned}
p & =\left[Y_{+} \eta^{2}\left(\frac{\mu_{0}^{\prime}(\bar{c})}{\mu_{0}^{\prime}(\bar{c})+2 \eta^{2} Y_{+}}\right)^{2}+\frac{1}{2} \mu_{0}^{\prime}(\overline{\bar{c}})\left(\frac{2 \eta^{2} Y_{+}}{\mu_{0}^{\prime}(\bar{c})+2 \eta^{2} Y_{+}}\right)^{2}\right]\left(c_{+}-c_{-}\right)^{2} \\
& =Y_{+}^{*} \eta^{2}\left(c_{+}-c_{-}\right)^{2}
\end{aligned}
$$

where

$$
Y_{+}^{*}:=\frac{1+2 \eta^{2} Y_{+} \mu_{0}^{\prime}(\overline{\bar{c}}) / \mu_{0}^{\prime}(\bar{c})^{2}}{\left[1+2 \eta^{2} Y_{+} / \mu_{0}^{\prime}(\bar{c})\right]^{2}} Y_{+}
$$

with $\bar{c}$ and $\overline{\bar{c}}$ lying between $c_{-}$and $c(v t+0)$ and therefore (by (32)) quite close to $c_{-}$. For isotropic elasticity, our result $(39,40)$ agrees with Hillert's (1) apart from the factor multiplying $Y_{+}$on the right of (40). The correction terms $2 \eta^{2} Y_{+} \mu_{0}^{\prime}(\overline{\bar{c}}) / \mu_{0}^{\prime}(\bar{c})^{2}$ and $2 \eta^{2} Y_{+} / \mu_{0}^{\prime}(c)$ are small under the dilute solution conditions envisaged by Hillert, although, for the reasons noted in the discussion of eqn (27), they can be as large as 0.2 under some conditions.

\section{Interpretation of the force}

According to the first line of (39), the driving pressure (force per unit area) on the grain boundary is the sum of two contributions; the first is due to the decrease in elastic energy density when the grain boundary moves, and the second (a relatively small correction when $2 \eta^{2} Y_{+} \mu_{0}^{\prime}(\overline{\bar{c}}) / \mu_{0}^{\prime}(\bar{c})^{2}$ is small) can be thought of as an osmotic pressure associated with the small difference in composition between the two sides of the grain boundary. Hillert [10] calls this force "the chemical driving force caused by a discontinuity in composition at the grain boundary". 
The elastic contribution to the force is not an elastic force of the usual kind: the $i$ th component of the elastic force across any plane $x_{3}=$ const. is $t_{3 i}$ which as we have seen is zero. However, the grain boundary can be thought of an interface between two different phases; the "phase" behind the moving grain boundary, which has the lower elastic energy per unit volume, grows at the expense of the one in front, which has the higher elastic energy. This is analogous to the growth of (say) an ice crystal in supercooled liquid water, where the phase with the lower free energy per unit volume grows at the expense of the other one.

Another way of thinking about the elastic force on the grain boundary makes use of Eshelby's "energy-momentum" tensor [15]. He defines this tensor as

$$
P_{i j}=w \delta_{i j}-\sum_{k} t_{k j} \partial u_{i} / \partial x_{k}
$$

It has the property that if $S$ denotes any closed surface with outward normal $n_{j}$ then the integral

$$
F_{i}=\sum_{j} \int_{S} P_{i j} n_{j} d S
$$

is the $i$ th component of the total force exerted by the elastic medium on the dislocations and other lattice defects (including misfitting solute atoms) enclosed by $S$. Let the surface in (42) be a rectangular box with faces in planes parallel and perpendicular to the grain boundary. The contributions to the integral from opposite faces of the box which are perpendicular to the boundary will cancel out, since the integrand is independent of $x_{1}, x_{2}$ and the normal vectors on opposite faces are equal and opposite. As for the faces parallel to the boundary, the contribution of the sum in (41) to the integrals over both these faces is zero, because $t_{k 3}=0$ as shown just before (10); so all that remains is the contribution of the term $w \delta_{i j}$. The force on the crystal defects inside $S$ is therefore $A(w(a)-w(b))$ where $x_{3}=a, x_{3}=b$ are the 
planes containing the faces parallel to the grain boundary and $A$ is the area of each of these faces.

To find the elastic force on the grain boundary, take $a=v t+0, b=$ $v t-0$; then eqns (12) and (28) show that this force is

$$
A[w(v t+0)-w(v t-0)]=A Y_{+} \eta^{2}\left[c(v t+0)-c_{+}\right]^{2}
$$

agreeing precisely with the term containing $Y_{+}$in our expression (37) for the total force. The other term in (37) represents the "chemical" contribution to the driving force, due to the difference in concentration across the grain boundary; this force, not being of elastic origin, is not included in Eshelby's formula.

\section{Discussion}

This paper uses a continuum theory to model the diffusive flow and the elastic deformations in the crystal ahead of the moving grain boundary. The grain boundary itself is modelled as an infinite plane moving with constant speed. To justify these modelling assumptions, it is necessary that the length scale for variations of composition and elastic deformation in the crystal grains be much larger than the atomic spacing and the thickness of the grain boundary; in symbols this condition is

$$
D / v \gg \lambda
$$

where $D$ is the diffusivity in the crystal grains, $v$ is the speed of the grain boundary, and $\lambda$ is its thickness $\star \star \star \star$. Another necessary condition (see ref. [5]) is that the time for diffusion to

\footnotetext{
$\overline{\star \star \star}$ According to the data in table 2 of ref.[2], the quantity $D / v$ varies widely; in the $\mathrm{Cu}-\mathrm{Ni}$ system it can be as small as about $10^{-12} \mathrm{~m}$ at temperature $550^{\circ} \mathrm{C}$ or below, but as large as $10^{-6} \mathrm{~m}$ at $900^{\circ} \mathrm{C}$, whereas $\lambda$ is of order $10^{-9} \mathrm{~m}$. Thus it appears that the condition (44) is satisfied in some DIGM experiments, particularly at high temperatures, but by no means all. Hillert [10] makes the same point.
} 
equalize the compositions on the two sides of the grain boundary should be much less than the time for the grain boundary to pass on, i.e. $\lambda^{2} / D_{g b} \ll \lambda / v$ where $\lambda$ is the thickness of the grain boundary and $D_{g b}$ the diffusivity within it. This last condition is equivalent to $D_{g b} / v \gg \lambda$. Since the diffusivity is much smaller in the crystal grains than in the grain boundary (indeed, in ref. [5]) the diffusivity in the crystal grains was taken to be zero), we have $D \ll D_{g b}$, so that $D_{g b} / v$ is inevitably $\gg \lambda$ if (44) is satisfied. The case where (44) does not hold is discussed in [3].

At the same time, to justify neglecting space derivatives in directions tangential to the grain boundary, the length scale for variations in a direction normal to the grain boundary should be much smaller than the length scale for tangential variations. An upper bound on this latter length scale is the thickness of the specimen, denoted here by $h$, so that one requirement is

$$
D / v \ll h
$$

In general, however, the length scale for tangential variations will be smaller than $h$. An estimate of this (potentially) smaller length scale can be obtained from the equation for diffusion in the grain boundary, according to which $D_{g b} \lambda d^{2} c_{-} / d s^{2} \sim\left(c_{+}-c_{-}\right) v$ (the dimensional version of eqn (1) of ref. [19]); the length scale for tangential composition variations in the grain boundary is therefore $\left(D_{g b} \lambda / v\right)^{1 / 2}$ and the condition for it to be much greater than the length scale for variations in the normal direction is

$$
D / v \ll\left(D_{g b} \lambda / v\right)^{1 / 2} .
$$

Eqns (44), (45) and (46) can be combined in a single formula,

$$
\max \left(\frac{D^{2}}{D_{g b} \lambda}, \frac{D}{h}\right) \ll v \ll \frac{D}{\lambda}
$$

We have already noted that $D \ll D_{g b}$, and so $D^{2} / D_{g b} \lambda \ll D / \lambda$; moreover since the specimen is always much thicker than the 
grain boundary we have $D / h \ll D / \lambda$; so there is always a wide range of speeds $v$ over which the theory in this paper can be used.

It is sometimes argued $[1,28,29]$ that DIGM does not require any specific driving mechanism, the decrease in free energy due to the changes in concentration that accompany the motion being a sufficient explanation. Against this, Balluffi and Cahn have argued [30] that this explanation, if correct, gives the implausible prediction that DIGM can occur even when the two species of atom are isotopes of one another. The present theory is consistent with the argument of Balluffi and Cahn, predicting that a mixture of isotopes would not exhibit DIGM, since the 'Vegard' coefficient $\eta$ in the formula (39) for the driving force would be zero. It might seem that the non-elastic 'chemical' term in the formula (37) could still give rise to a force on the grain boundary even though the elastic term is zero, but in fact the non-elastic term is now zero as well because, in the absence of any elastic contribution to the diffusion potential, the mole fractions are the same on the two sides of the grain boundary (see eqn (34)).

Besides predicting the driving force for steady-state DIGM, the present theory also gives a prediction about the direction in which DIGM will start out, given an initially stationary grain boundary. The mechanism is described by Sutton and Balluffi [4]: "Solute atoms initially diffuse into the boundary. They then begin to penetrate into the two adjoining crystals. ... coherency stresses are generated in the thin diffused layers ... if they remain coherent with the bulk. If the boundary layer is asymmetric ... the boundary layer will be urged to move towards the crystal with the higher strain energy in order to consume it. Once begun, this process can continue in a self-sustaining manner." To make this description quantitative, suppose that the material is initially unstressed on both sides of the grain boundary, at solute concentration $c_{\infty}$ and diffusion potential $\mu_{0}\left(c_{\infty}\right)$. Now let the diffusion potential in the grain boundary be changed to a new value $\mu_{g b}$. In each grain the diffusion potential now varies from $\mu_{g b}$ at the grain boundary to $\mu_{0}\left(c_{\infty}\right)$ at large distances from it; the resulting 
gradients of diffusion potential cause material to diffuse, bringing about elastic misfit strains on both sides of the grain boundary. Making the plane $x_{3}=0$ the initial position of the grain boundary, with an arbitrary choice for the positive direction along the $x_{3}$ axis, and then carrying out a calculation like the one that led to (37), it can be shown that the force per unit area on the grain boundary before it has started to move is

$$
p=\left(Y_{+}^{* *}-Y_{-}^{* *}\right) \eta^{2}\left(c_{g b}-c_{\infty}\right)^{2}
$$

where $c_{g b}$ is defined by $\mu_{g b}=\mu_{0}\left(c_{g b}\right)$. Here the definition of $Y_{+}^{* *}$ is like that of $Y_{+}^{*}$, eqn (40), but with $c_{-}$and $c(v t+0)$ changed to $c_{\infty}$ and $c_{g b}$ in the definitions of $\bar{c}$ and $\overline{\bar{c}}$; the definition of $Y_{-}^{* *}$ is like that of $Y_{+}^{* *}$ but with $Y_{+}$replaced by $Y_{-}$. If the crystal is anisotropic, with differently oriented crystal axes in the two grains, then $Y$, as defined in eqn (13), may take different values on the two sides; there will then be an elastic force pushing the grain boundary towards the grain with the larger value of $Y$, as in Sutton and Balluffi's explanation. A nice experiment of Liang and King [22] using hexagonal Zn-Cd bicrystals confirms that DIGM starts if and only if the crystal axes and the grain boundary are oriented so as to make $Y$ different in the two grains.

Once the motion has started, one expects the stress in the newly grown piece of crystal to be small, except in a region near the original position of the grain boundary; so by the time the grain boundary has travelled a distance comparable to the thickness of the specimen, the retarding effect of the elastic deformation in the growing grain will have begun to die away and the force will eventually be given by the steady-state formula (39) which involves the elastic constants of the shrinking grain only.

If the grain boundary is not straight initially, the local direction of the normal vector $\mathbf{n}$ varies with position and so the local value of $Y_{+}^{* *}-Y_{-}^{* *}$, which depends on $\mathbf{n}$ (see eqn (14)), also varies with position (a possibility mentioned in ref.[22]) and may even have different signs in different places. As a result the initial direction 
of motion may be different at different places on the same grain boundary. Indeed some experiments do show different directions of motion on different parts of the same grain boundary; for an example see Fig 9 of ref. [2].

The mechanism for DIGM discussed here is akin to the mechanism for liquid-film migration (LFM) proposed in 1984 by Yoon, Cahn, Handwerker, Blendell and Baik [31,32], but there are important differences. LFM is a phenomenon similar to DIGM but with a layer of liquid between the two crystal grains. Yoon et al. assume, just as in the present paper, that diffusion just ahead of the advancing liquid film sets up a stress field whose effect on the chemical potentials must be allowed for; but there are now two interfaces, at each of which both chemical potentials, rather than just their difference, must be continuous. Moreover, the role of diffusion in controlling the speed of migration is also quite different: for LFM, the controlling factor is the rate at which atoms can cross from one face of the film to the other, whereas for DIGM it is the rate at which they can be transported by lateral diffusion from one part of the grain boundary to another (and, ultimately, to or from the environment of the specimen).

\section{Acknowldgements}

I am indebted to John Cahn for many years' patient teaching about DIGM, to John Ockendon and the late Jack Christian for penetrating questions which led to the writing of this paper, to Augusto Visintin, the local organizer of the conference (Trento 2002) where some of those questions were asked, and to a helpful referee for telling me about anisotropic misfit strains and ref. [22].

\section{References}

[1] Hillert, M. and Purdy, G. R., Chemically induced grain boundary migration, Acta Metall. (1978) 26 333-340. 
[2] Handwerker, C., "Diffusion-Induced Grain Boundary Migration in thin Films", pp. 245-322 of Diffusion Phenomena in Thin Films and Microelectronic Materials, ed. D. Gupta and P. S. Ho, Noyes Publications, Park Ridge, NJ (1988).

[3] Yoon, D. Y., Theories and observations of chemically induced interface migration, International Materials Reviews (1995) 40 149-179.

[4] Sutton, A.P. and Balluffi, R.W. Interfaces in crystalline materials, 516-7, Oxford (1995).

[5] Cahn, J. W., Fife, P. C. and Penrose, O., A phase-field model for diffusioninduced grain boundary motion, Acta Mater. (1997) 45 4397-4413.

[6] Lee, K. R., Baik Y. J. and Yoon D. N., A critical test for the coherency strainenergy as the driving force for the discontinuous precipitation in Mo-Ni alloy, Acta Metall. Mater. (1987) 35 2145-2150.

[7] Rhee, W.-H. and Yoon D.N., The grain-boundary migration induced by diffusional coherency strain in Mo-Ni alloy, Acta Metall. (1989) 37 221-228.

[8] Sulonen, M.S., Discontinuous mode of dissolution of a $\beta$-phase precipitate into a Cu-Cd solution, Acta Metall. (1960) 8 669-676.

[9] Sulonen, M.S., On the driving force of discontinuous precipitation and dissolution. Acta Metall. (1964) 12 749-753.

[10] Hillert, M., On the driving force for diffusion induced grain-boundary migration. Scripta Met. (1983) 17 237-240.

[11] Cahn, J. W., On spinodal decomposition, Acta metall. (1961) 9 795-801.

[12] Larché, F. C. and Cahn, J. W., A linear theory of thermochemical equilibrium of solids under stress, Acta Metall. (1978) 21 1051-1063.

[13] Larché, F. C. and Cahn, J. W., Thermomechanical equilibrium of multiphase solids under stress, Acta Metall. (1978) 26 1579-1589.

[14] Larché, F. C. and Cahn, J. W., The effect of self-stress on diffusion in solids, Acta Metall. (1982) 30 1835-1845.

[15] Eshelby, J. D., The elastic energy-momentum tensor, Journal of Elasticity (1975) 5 321-335.

[16] Fife, P. C., Cahn, J. W. and Elliott, C. M., A free-boundary model for diffusioninduced grain boundary motion, Interfaces Free Bound. (2001) 3 291-336.

[17] Deckelnick, K., Elliott, C. M. and Styles, V. Numerical diffusion-induced grain boundary motion, Interfaces Free Bound. (2001) 3 393-414.

[18] Brener, E. A. and Temkin, D. E., Theory of discontinuous precipitation: importance of the elastic strain, Acta Mater (2003) 51 797-803. 
[19] Penrose, O. and Cahn J. W., A mathematical model for diffusion-induced grain boundary motion, Birkhäuser International Series of Numerical Mathematics (ISNM) (2003) 147 (Proceedings of the conference on Free Boundary Problems, Trento 2002, ed. A. Visintin, P. Colli and C. Verdi) 237-254.

[20] Fratzl, P., Penrose, O. and Lebowitz, J., Modeling of phase separation in alloys with coherent elastic misfit, J. Stat. Phys. (1999) 95 1429-1503.

[21] Landau, L.D. and Lifshits, E.M., Theory of elasticity (translated by J. B. Sykes and W. H. Reid), Pergamon, London, 1959.

[22] Liang, L. and King, A. H., Diffusion induced grain boundary migration in the zinc-cadmium system, Acta mater. (1996) 44 2983-2998.

[23] Khachaturyan, A. G. Theory of structural transformations in solids. Wiley, New York (1983).

[24] Cahn, J. W., On spinodal decomposition in cubic crystals, Acta metall. (1962) 10 179-183.

[25] Khachaturyan, A. G., Some questions concerning the theory of phase transformations in solids, Sov. Phys. Solid State (1967) 8 2163-2168. Translated from Fiz. Tverd. Tela (1966) 8 2710-2717.

[26] Hilliard, J. E. pp 497-560 of Phase Transformation, papers presented at a seminar on 12 and 13 October 1968. American Society for Metals, Metals Park, Ohio (1970).

[27] Cahn, J. W. and Balluffi, R. W., On diffusional mass transport in polycrystals containing stationary or migrating grain boundaries, Scripta Met. (1979) 13 499502 .

[28] Klinger, L. K., Brechet, Y. J. M. and Purdy, G. R., On velocity and spacing selection in discontinuous precipitation: I simplified analytical approach, Acta Mater. (1997) 45 5005-5013.

[29] Kajihara, M. and Gust, W., Driving force for grain boundary migration during alloying by DIGM and DIR in binary systems, Scripta Mater. (1998) 38 16211627 .

[30] Balluffi, R. W. and Cahn, J. W., Mechanism for diffusion induced grain boundary migration, Acta Met. (1981) 29 493-500.

[31] Handwerker, C. A., Cahn, J. W., Yoon D.N. and Blendell J.E., "The effect of coherency strain on alloy formation: migration of liquid films", pp 275-322 of Diffusion in solids: recent developments, ed. M A Dayanda and G E Murch, The Metallurgical Society (1985).

[32] Yoon, D. N., Cahn, J. W., Handwerker C. A., Blendell, J. E. and Baik, Y. J., "Coherency strain induced migration of liquid films through solids", pp 19-31 of Interface Migration and Control of Microstructure, ed. C. S. Pande, American Society for Metals (1986). 